\section{Cureus}

Received 12/10/2018

Review began 01/22/2019

Review ended 02/19/2019

Published 03/01/2019

\section{(c) Copyright 2019}

Gray et al. This is an open access article distributed under the terms of the Creative Commons Attribution License CC-BY 3.0., which permits unrestricted use, distribution, and reproduction in any medium, provided the original author and source are credited.

\title{
Hypopituitarism After Traumatic Brain Injury
}

\author{
Sanjiv Gray ${ }^{1}$, Tracy Bilski ${ }^{2}$, Beatrice Dieudonne ${ }^{3}$, Saqib Saeed ${ }^{3}$ \\ 1. Surgery, University of Central Florida, Orlando, USA 2. Surgery, University of South Florida Morsani \\ College of Medicine, Kissimmee, USA 3. Surgery, Harlem Hospital Center, New York, USA
}

$\square$ Corresponding author: Sanjiv Gray, sanjivfg@gmail.com

Disclosures can be found in Additional Information at the end of the article

\section{Abstract}

Acquired hypopituitarism is associated with traumatic brain injury. This neuroendocrine dysfunction can cause both short-term and long-term morbidity resulting in a cognitive, physiological, and behavioral decline, which increases the burden of the disease and the cost of care. Data in the trauma literature is derisory on this subject. The aim of this review is to edify clinicians on this condition, outline the screening criteria and methods for hypopituitarism after traumatic brain injury, and bring awareness to the chronic effects.

Categories: Endocrinology/Diabetes/Metabolism, General Surgery, Trauma

Keywords: hypopituitarism, traumatic brain injury, head injury, neuroendocrine dysfunction, adrenal insufficiency, diabetes insipidus, central diabetes insipidus

\section{Introduction And Background}

Traumatic brain injury (TBI) has a significant impact on our population with an incidence of approximately 2.8 million cases per year in the United States and approximately 50,000 deaths annually. After exsanguination, TBI has been found to be the leading cause of death in the trauma population; among the survivors, 80,000 - 90,000 will experience the onset of long-term disability [1]. The leading causes of TBI are falls (54-79\%), being struck by or against an object (15\%), and motor vehicle collisions (15\%). Between 2007 and 2013, TBI-related emergency department visits increased by $47 \%$, hospitalization rates decreased by $2.5 \%$, and death rates decreased by $5 \%$ [1]. Most cases are mild but approximately $20-40 \%$ are classified as moderate or severe. With improvements in the trauma system, including prehospital care, trauma resuscitation, and development of TBI protocols, more patients are surviving to discharge. These survivors often have physical, cognitive, and behavioral alterations. Acute hypopituitarism can affect the patient's hemodynamics and can have an influence on the longterm recovery from the TBI. This review aims to highlight an increasingly recognized entity, which can have an impact on the functional outcomes of TBI.

\section{Review}

Cyran et al. [2] first described hypopituitarism after TBI in 1918, and in recent years, there have been multiple descriptions in the neurology, endocrine, and physiatry literature.

Clinicians need to be aware of these post-TBI endocrinopathies and provide appropriate referral to an endocrinologist or internist for further testing and management. Hypopituitarism is underdiagnosed in the TBI population as signs and symptoms are vastly non-specific in these critically ill trauma patients. Pituitary failure was previously thought to be rare, but recent literature reviews show the prevalence of hypopituitarism ranges from $15 \%$ to $90 \%$ [3-8]. In a systematic review, Schneider et al reported a pooled prevalence of $27.5 \%$ for hypopituitarism 
after TBI with the prevalence of anterior pituitary dysfunction ranging from $15 \%$ to $68 \%$ across all studies in the review [8]. This would make TBI the most common cause of acquired hypopituitarism. Several factors contribute to the pathophysiology of hypopituitarism after TBI, such as a direct pituitary gland/stalk injury or ischemia/infarction of the pituitary gland [910], which can be attributed to venous infarction of the long hypophyseal portal veins. Secondary insults arise from hypoxia, hypotension, anemia, raised intracranial pressure, and reduced cerebral perfusion pressure. Tanriverdi et al. suggest that autoimmunity may cause neuroendocrine dysfunction with the presence of anti-pituitary antibodies up to three years after diagnosis [11-12]. Autoimmunity may result from the disruption of the blood-brain barrier, which exposes brain proteins to the systemic circulation. Magnetic resonance imaging (MRI) is the preferred method to assess the pituitary gland post-injury [13].

\section{Understanding pituitary hormonal function}

Understanding the function of the various pituitary hormones will aid the clinician in screening for symptoms of deficiency of specific hormones. Growth hormone $(\mathrm{GH})$ is important for microtubular regeneration, lipid metabolism, and dendritic growth and regrowth. Insulin-like growth factor 1 (IGF-1) affects the functional use of glucose in the brain. IGF-1 depletion causes disruption in lipid and microtubule metabolism, leading to impaired neuronal, somatic, and dendritic growth. Functions mediated by GH receptors in the hippocampal area may be involved in the hormone's action on memory and cognitive function [14]. Adrenocorticotropic hormone (ACTH) and cortisol deficiency result in fatigue, weakness, and inability to respond to physiological stress. In addition, mood disorders, decreased memory, and frank psychosis can be seen with chronic cases of cortisol deficiency. Decreases in thyroid function lead to a decrease in the basal metabolic rate. This combination could lead to symptoms of physical fatigue. Follicle-stimulating hormone (FSH) and luteinizing hormone (LH) play integral parts in the production of the sex hormones, estrogen, and testosterone. Besides the effects on reproduction, menses, libido, fertility, pregnancy, and maintenance of sexual characteristics, deficiencies in these hormones have also been linked to decreases in bone and muscle mass $[15]$.

The degree of hypopituitarism is determined by the number of axes involved. Growth hormone deficiency is the most commonly reported deficiency in severe and moderate TBI with a prevalence of $2 \%$ to $66 \%[4,6,16]$. Adrenal deficiency ranges from 0 to $60 \%$, hypothyroidism from 0 to $29 \%$, central hypogonadism from 0 to $29 \%$, and hyperprolactinemia from 0 to $48 \%$. The wide ranges and variability are explained by the lack of screening guidelines, different intervals from TBI occurrence to screening, varying severity of head injury, different testing methods, and different study designs [17]. Most of the literature includes case studies, retrospective studies, and cross-sectional studies. Adrenal insufficiency (AI) and central diabetes insipidus (DI) are common in the acute phase but tend to resolve. Hyperprolactinemia cases are mild and are typically clinically insignificant [18].

\section{Screening and diagnosis}

The optimal time for screening is still debatable as pituitary dysfunction during the acute phase of TBI to three weeks does not necessarily lead to long-term hypopituitarism [8]. Schneider et al. showed that hypopituitarism is found in 56\% of TBI patients at three months but only in $36 \%$ at one year. The prevalence of hypopituitarism in Schneider's series for cases of severe, moderate, and mild TBI were 35.3\%, 10.9\%, and 16.8\%, respectively [19]. In addition, trauma patients have hormonal changes and systemic inflammatory response syndrome with findings similar to pituitary failure. There is also increased cortisol, growth hormone, prolactin, and vasopressin as part of the stress response and acute adaptive response to injury [20]. These levels are also affected by medications, surgery, and drug intoxication. Pituitary function during this time is noted to be variable with either worsening or improving function. This has been 
attributed to the revascularization of the cellular pituicyte [21]. In a consensus statement, Ghigo et al. maintained that routine basal hormonal testing should be performed on any patient who has been hospitalized with a TBI and has symptoms, such as hyponatremia and hypotension, to rule out adrenal insufficiency, which can be life-threatening (Figure 1) [22]. They also recommended that patients who sustained a mild TBI and are symptomatic or admitted greater than 24 hours and those with moderate and severe TBI should undergo a baseline hormonal evaluation at three and 12 months post-head injury. All post-TBI patients with any signs or symptoms of hypopituitarism should undergo hormonal testing without further delay, even if greater than one year from the injury, as it is unlikely that any hormonal deficit would be transient after that period of time. Lorenzo et al. [23] proposed screening at one year after TBI, especially for gonadal and somatotropic axes, while acutely replacing other needed hormones.

Figure 1. Acute Phase Screening For Hypopituitarism After Traumatic Brain Injury

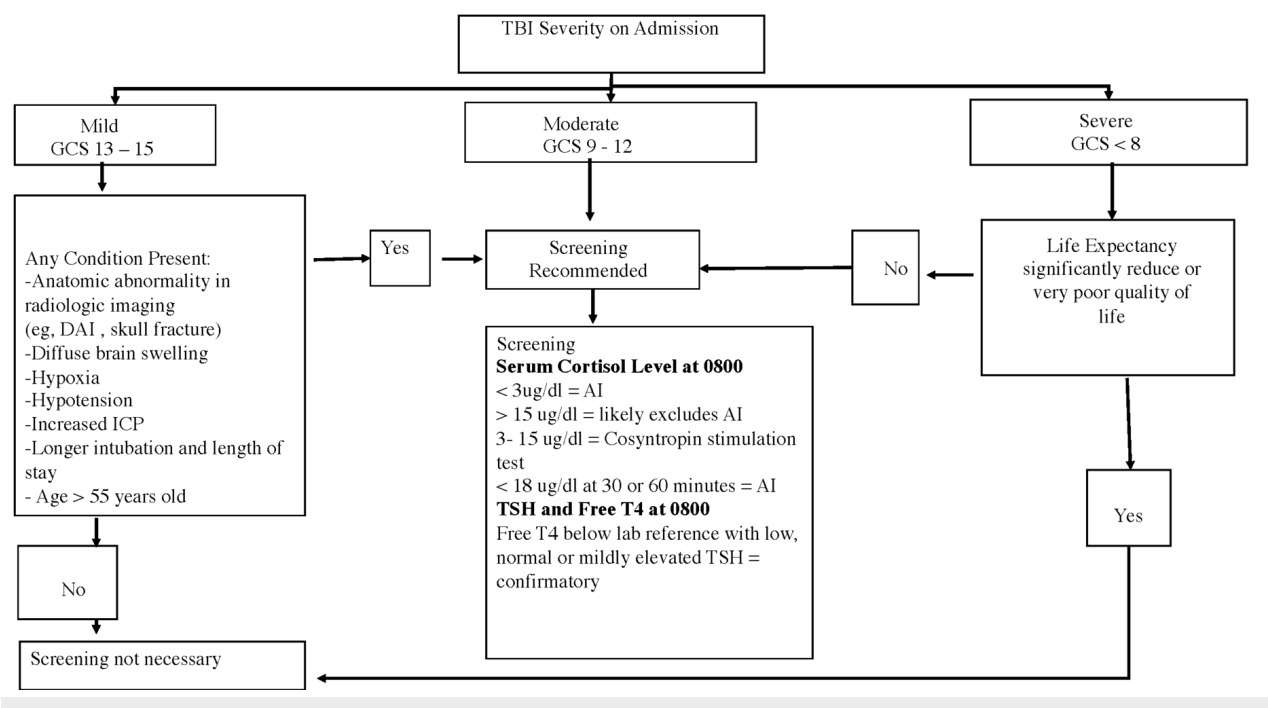

\section{FIGURE 1: Acute Phase Screening for Hypopituitarism After Traumatic Brain Injury}

Al - adrenal insufficiency; DAI - diffuse axonal injury; GCS - Glasgow Coma Scale; ICP - intracranial pressure; TBI - traumatic brain injury; TSH - thyroid stimulating hormone; ug/dl - microgram per deciliter

Recommendations of an expert panel assembled by the Defense Centers of Excellence (DCoE) for Psychological Health and Traumatic Brain Injury to address neuroendocrine dysfunction in mild TBI (Glasgow Coma Scale (GCS): 12-15) was released in December 2012 to encourage primary care providers to consider screening patients with persistent symptoms after TBI and even up to 36 months post-injury [24]. Other studies have shown that ongoing dynamic hormonal changes persist up to three years in mild TBI.

Patients in a persistent vegetative state [25] or with a low life expectancy will not benefit from hormone replacement; therefore, screening is not recommended. In addition, some patients with mild TBI or repetitive head injury maybe develop isolated hypopituitarism; therefore, vigilance is needed [26].

To summarize, TBI patients who required hospitalization for at least 24 hours, those with an abnormality on the initial head computed tomography (CT), and those who have signs and 
symptoms of pituitary failure after TBI should be screened at three months and one year postinjury and even further out if symptomatic (Figure 2). All symptomatic patients should be screened immediately. Consultation with an endocrinologist is recommended for added expertise and the management of hormone replacement therapy if required.

Figure 2. Follow up Screening For Hypopituitarism After Traumatic Brain Injury

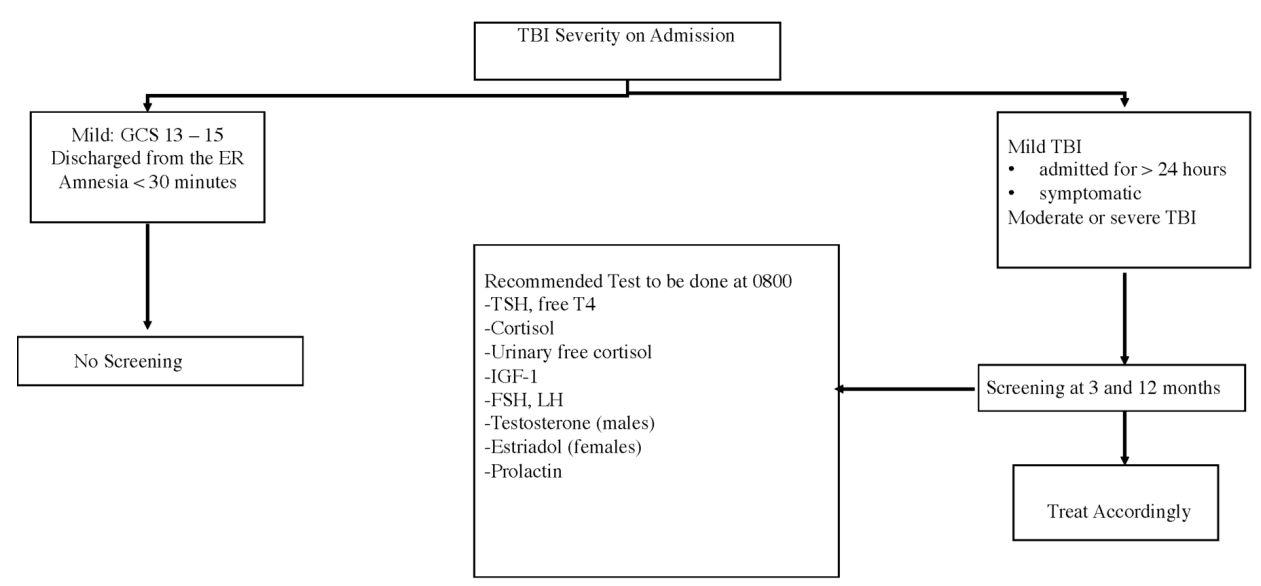

FIGURE 2: Follow-up Screening for Hypopituitarism After Traumatic Brain Injury

Al - adrenal insufficiency; DAI - diffuse axonal injury; FSH - follicle stimulating hormone; GCS Glasgow Coma Scale; ICP - intracranial pressure; IGF-1 - insulin-like growth factor 1; LH luteinizing hormone; TBI - traumatic brain injury; TSH - thyroid stimulating hormone; ug/dl microgram per deciliter

\section{Predictors of neuroendocrine dysfunction}

In a retrospective study, Silva et al. [27] showed that post-traumatic seizures, intracranial hemorrhage, petechial brain hemorrhage, and focal cortical contusion are predictors of central adrenal insufficiency. Motor vehicle crash (MVC), as a mechanism for TBI, was a risk factor for both thyroid stimulating hormone (TSH) and ACTH deficiencies. Hospitalization after TBI and the presence of intracranial hemorrhage on imaging showed trends towards being risk factors for GH deficiency. Other conditions associated with hypopituitarism are advanced age, increased intracranial pressure, prolonged intubation greater than 10 days, diffuse axonal injury, basal skull fractures, hypoxia, hypotension, and the duration of coma. Cranial vault fracture was found to correlate negatively [22, 27-28]. In a study by Schneider et al., patients with DI were primarily victims of MVCs and had brain MRI showing intracranial hemorrhage, subarachnoid hemorrhage, or focal cortical contusion [28]. They concluded that TBI patients who have experienced an MVC and/or those with post-traumatic seizures, intracranial hemorrhage, petechial brain hemorrhages, and/or focal cortical contusions are at high risk for neuroendocrine dysfunction, including central adrenal insufficiency and DI, and should be referred for pituitary hormone testing. The predictors appear to be applicable to most TBI patients and hence, the broad recommendation to screen most patients.

The Endocrine Society Clinical Practice Guidelines published in 2016 provides recommendations for the diagnosis of hypopituitarism [29]. The guideline recommends measuring serum cortisol levels at 8 - 9 am as the first-line test for 
diagnosing central adrenal insufficiency (AI). A cortisol level $<3 \mu \mathrm{g} / \mathrm{dL}$ is indicative of AI and a cortisol level $>15 \mu \mathrm{g} / \mathrm{dL}$ likely excludes an AI diagnosis. A corticotropin stimulation test can be performed to diagnose AI when the morning cortisol values are between $3 \mu \mathrm{g} / \mathrm{dLand}$

$15 \mu \mathrm{g} / \mathrm{dL}$. Peak cortisol levels $<18.1 \mu \mathrm{g} / \mathrm{dL}$ at 30 minutes or 60 minutes indicate AI. For central hypothyroidism, a free T4 level below the laboratory reference range in conjunction with a low, normal, or mildly elevated TSH in the setting of pituitary disease is usually confirmatory. For GH deficiency (GHD), GH stimulation testing is recommended as single GH measurements are not helpful. Appropriately controlled body mass index (BMI) cutoffs to assess peak GH values should be done. Biochemical testing for GHD in patients with clear-cut features of GHD and other documented pituitary hormone deficits should not be done. The stimulation test can be the insulin tolerance test, the glucagon stimulation test, the GH-releasing hormone (GHRH) arginine test, or GHRH/GH releasing peptide-6 (GHRP-6) test. The GHRH/GHRP-6 test is noted to be reliable and is not affected by factors which modify $\mathrm{GH}$ secretions. In males with suspected hypogonadism, it is recommended to measure the serum testosterone, FSH, and LH in the absence of acute/subacute illness and before $10 \mathrm{am}$ (after an overnight fast) combined with a serum prolactin (PRL) level. In the presence of oligomenorrhea or amenorrhea, it is recommended to measure serum estradiol (E2), FSH, and LH. Clinicians should exclude other causes of menstrual irregularities related to impaired ovulation. For postmenopausal women, the absence of high serum FSH and LH is sufficient for a diagnosis of gonadotrope dysfunction (provided the patient is not on hormone replacement therapy (HRT)). To assess for central diabetes insipidus, serum and urine osmolarity should be measured simultaneously in patients with polyuria (more than $50 \mathrm{~mL} / \mathrm{kg}$ of body weight/24 hours, 3.5 liters/day (L/d) in a $70-\mathrm{kg}$ person). In the presence of high serum osmolarity (> $295 \mathrm{mOsmol} / \mathrm{L})$, urine osmolarity should reach approximately $600 \mathrm{mOsmol} / \mathrm{L}$ (urine osmolality/plasma osmolality ratio should be $\geqslant 2$ ), whereas the urine dipstick should be negative for glucose. The involvement of an endocrinologist is recommended to ensure that appropriate testing and follow-up is provided [29]. In addition, hospital discharge information should include the recommendation for the screening by the patient's primary care provider.

In the chronic phase, post-TBI hypopituitarism can manifest as lethargy, poor sleep, inattention, difficulty concentrating, memory impairment, anxiety, poor judgment, depression, irritability, insomnia, and diminished libido [23]. Wachter et al. [30] found that neuropsychological and quality of life (QoL) deficits were associated more frequently with hemorrhagic lesions on CT scans than with hypopituitarism.

\section{Management and outcomes}

Hypopituitarism contributes to TBI-related mortality and functional and cognitive morbidity. GH deficiency has the most effect on the outcome [31-32]. There is a clear association between pituitary dysfunction and adverse cognitive outcomes, such as memory, attention, language, physical conditioning, and mood disorders. It can also lead to dyslipidemia, hypertriglyceridemia, adiposopathy, insulin resistance, reduced quality of life, and increased risk of premature cardiovascular death from endothelial dysfunction and atherosclerosis [33]. These findings are independent of the BMI of the patient. It may also limit recovery and rehabilitation [34], leading to chronic disability. There is reported improvement in the QoL after GH replacement in post-TBI hypopituitarism, as well as improvement in cognitive impairments and functional independence measures. Adrenal crisis after TBI can be treated with glucocorticoid replacement [35-41]. Testosterone replacement in hypogonadal men is associated with decreased anger and irritability and increased libido and energy. Replacement of estrogen has been shown to improve verbal memory, reasoning, vigilance, and motor speed in symptomatic postmenopausal females. Patients with post-TBI pituitary dysfunction may receive suboptimal rehabilitation unless the underlying hormone deficiency is identified and treated. Replacement is also important in these post-traumatic patients who maybe require further surgical intervention and are at risk for adrenal insufficiency during the postoperative 
period. Once identified, TSH, antidiuretic hormone (ADH), and ACTH deficiencies should be replaced, but GH replacement therapy in adults in the setting for hypopituitarism is controversial. Therefore, referral to an endocrinologist is recommended for individualized management of the various deficiencies.

\section{Conclusions}

Clinicians involved in the management of TBI patients should consider hypopituitarism and its impact on long-term morbidity. Evaluation of pituitary function on an ongoing basis after TBI is paramount irrespective of the severity of the initial injury. Endocrinology and physiatry involvement for specialized testing and long-term follow-up is recommended for all TBI patients who were hospitalized and/or are symptomatic. Hormonal replacement is essential for optimal rehabilitation for TBI patients with a positive screen. Continuing medical education will increase physician awareness, and informing patients and their families will help to foster better follow-up. Further studies are needed to determine the true incidence of hypopituitarism, define the pathophysiology of hypopituitarism after TBI, and refine the predictors of hypopituitarism and the effects of pituitary hormone replacement on the metabolic profile, psychosocial issues, and neurocognitive behaviors.

\section{Additional Information}

\section{Disclosures}

Conflicts of interest: In compliance with the ICMJE uniform disclosure form, all authors declare the following: Payment/services info: All authors have declared that no financial support was received from any organization for the submitted work. Financial relationships: All authors have declared that they have no financial relationships at present or within the previous three years with any organizations that might have an interest in the submitted work. Other relationships: All authors have declared that there are no other relationships or activities that could appear to have influenced the submitted work.

\section{References}

1. Centers for Disease Control and Prevention Division of Unintentional Injury Prevention . (2017). Accessed: July 24, 2018: http://www.cdc.gov/traumaticbraininjury/get_the_facts.html.

2. Cyran E: Hypophysenschädigung durch Schädelbasisfraktur. Dtsch Med Wochenschr. 1918, 44:1261.

3. Fernandez-Rodriguez E, Bernabeu I, Castro AI, Kelestimur F, Casanueva FF: Hypopituitarism following traumatic brain injury: determining factors for diagnosis. Front Endocrinol (Lausanne). 2011, 2:25. 10.3389/fendo.2011.00025

4. Aimaretti G, Ghigo E: Traumatic brain injury and hypopituitarism. ScientificWorldJournal. 2005, 5:777-81. 10.1100/tsw.2005.100

5. Lieberman SA, Oberoi AL, Gilkison CR, Masel BE, Urban RJ: Prevalence of neuroendocrine dysfunction in patients recovering from traumatic brain injury. J Clin Endocrinol Metab. 2001, 86:2752-56. 10.1210/jcem.86.6.7592

6. Tanriverdi F, Senyurek H, Unluhizarci K, Selcuklu A, Casanueva FF, Kelestimur F: High risk of hypopituitarism after traumatic brain injury: a prospective investigation of anterior pituitary function in the acute phase and 12 months after trauma. J Clin Endocrinol Metab. 2006, 91:2105-11. 10.1210/jc.2005-2476

7. Leal-Cerro A, Flores JM, Rincon M, et al.: Prevalence of hypopituitarism and growth hormone deficiency in adults long-term after severe traumatic brain injury. Clin Endocrinol (Oxf). 2005, 62:525-32. 10.1111/j.1365-2265.2005.02250.x

8. Schneider HJ, Kreitschmann-Andermahr I, Ghigo E, Stalla GK, Agha A: Hypothalamopituitary dysfunction following traumatic brain injury and aneurysmal subarachnoid hemorrhage: a systematic review. JAMA. 2007, 298:1429-38. 10.1001/jama.298.12.1429

9. Dusick JR, Wang C, Cohan P, Swerdloff R, Kelly DF: Pathophysiology of hypopituitarism in the 
setting of brain injury. Pituitary. 2012, 15:2-9. 10.1007/s11102-008-0130-6

10. Maiya B, Newcombe V, Nortje J, et al.: Magnetic resonance imaging changes in the pituitary gland following acute traumatic brain injury. Intensive Care Med. 2008, 34:468-75. 10.1007/s00134-007-0902-X

11. Tanriverdi F, De Bellis A, Bizzarro A, et al.: Antipituitary antibodies after traumatic brain injury: is head trauma-induced pituitary dysfunction associated with autoimmunity? Eur J Endocrinol. 2008, 159:7-13. 10.1530/EJE-08-0050

12. Tanriverdi F, Schneider HJ, Aimaretti G, Masel BE, Casanueva FF, Kelestimur F: Pituitary dysfunction after traumatic brain injury: a clinical and pathophysiological approach. Endocr Rev. 2015, 36:305-42. 10.1210/er.2014-1065

13. Makulski DD, Taber KH, Chiou-Tan FY: Neuroimaging in posttraumatic hypopituitarism. J Comput Assist Tomogr. 2008, 32:324-28. 10.1097/RCT.0b013e3181636ed4

14. Cheng CM, Mervis RF, Niu SL, et al.: Insulin-like growth factor 1 is essential for normal dendritic growth. J Neurosci Res. 2003, 73:1-9. 10.1002/jnr.10634

15. Elovic EP: Anterior pituitary dysfunction after traumatic brain injury, part I . J Head Trauma Rehabil. 2003, 18:541-43. 10.1097/00001199-200311000-00008

16. Aimaretti G, Ambrosio MR, Di Somma C, et al.: Residual pituitary function after brain injuryinduced hypopituitarism: a prospective 12-month study. J Clin Endocrinol Metab. 2005, 90:6085-92. 10.1210/jc.2005-0504

17. Kokshoorn NE, Wassenaar MJ, Biermasz NR, Roelfsema F, Smit JW, Romijn JA, Pereira AM: Hypopituitarism following traumatic brain injury: prevalence is affected by the use of different dynamic tests and different normal values. Eur J Endocrinol. 2010, 162:11-18. 10.1530/EJE-090601

18. Agha A, Phillips J, O'Kelly P, Tormey W, Thompson CJ: The natural history of post-traumatic hypopituitarism: implications for assessment and treatment. Am J Med. 2005, 118:1416.e11416.e7. 10.1016/j.amjmed.2005.02.042

19. Schneider HJ, Schneider M, Saller B, et al.: Prevalence of anterior pituitary insufficiency 3 and 12 months after traumatic brain injury. Eur J Endocrinol. 2006, 154:259-65.

10.1530/eje.1.02071

20. Klose M, Juul A, Struck J, Morgenthaler NG, Kosteljanetz M, Feldt-Rasmussen U: Acute and long-term pituitary insufficiency in traumatic brain injury: a prospective single-centre study. Clin Endocrinol (Oxf). 2007, 67:598-606. 10.1111/j.1365-2265.2007.02931.x

21. Benvenga S, Campenní A, Ruggeri RM, Trimarchi F: Hypopituitarism secondary to head trauma. J Clin Endocrinol Metab. 2000, 85:1353-61. 10.1210/jcem.85.4.6506

22. Ghigo E, Masel B, Aimaretti G, et al.: Consensus guidelines on screening for hypopituitarism following traumatic brain injury. Brain Inj. 2005, 19:711-24. 10.1080/02699050400025315

23. Lorenzo M, Peino R, Castro AI, et al.: Hypopituitarism and growth hormone deficiency in adult subjects after traumatic brain injury: who and when to test. Pituitary. 2005, 8:233-7. 10.1007/s11102-006-6046-0

24. Defense Centers of Excellence (DCoE) for Psychological Health and Traumatic Brain Injury . (2017). Accessed: September 17: https://dvbic.dcoe.mil/files/resources/DCoE_TBI_NED_Clnical_Recommendations.pdf.

25. Multi-Society Task Force on PVS: Medical aspects of the persistent vegetative state (1) . N Engl J Med. 1994, 330:1499-508. 10.1056/NEJM199405263302107

26. Hellawell DJ, Pentland B : Relatives reports of long term problems following traumatic brain injury or subarachnoid haemorrhage. Disabil Rehabil. 2001, 23:300-5.

10.1080/dre.23.7.300.305

27. Silva PP, Bhatnagar S, Herman SD, Zafonte R, Klibanski A, Miller KK, Tritos NA: Predictors of hypopituitarism in patients with traumatic brain injury. J Neurotrauma. 2015, 32:1789-95. 10.1089/neu.2015.3998

28. Schneider M, Schneider HJ, Yassouridis A, Saller B, Rosen FV, Stalla GK: Predictors of anterior pituitary insufficiency after traumatic brain injury. Clin Endocrinol (Oxf). 2008, 68:206-12. 10.1111/j.1365-2265.2007.03020.x

29. Fleseriu M, Hashim IA, Karavitaki N, Melmed S, Murad MH, Salvatori R, Samuels MH: Hormonal replacement in hypopituitarism in adults: an Endocrine Society Clinical Practice Guideline. J Clin Endocrinol Metab. 2016, 101:3888-921. 10.1210/jc.2016-2118

30. Wachter D, Gündling K, Oertel MF, Stracke H, Böker DK: Pituitary insufficiency after traumatic brain injury. J Clin Neurosci. 2009, 16:202-208. 10.1016/j.jocn.2008.01.009 
31. Kelly DF, McArthur DL, Levin H, et al.: Neurobehavioral and quality of life changes associated with growth hormone insufficiency after complicated mild, moderate, or severe traumatic brain injury. J Neurotrauma. 2006, 23:928-42. 10.1089/neu.2006.23.928

32. Bondanelli M, Ambrosio MR, Cavazzini L, et al.: Anterior pituitary function may predict functional and cognitive outcome in patients with traumatic brain injury undergoing rehabilitation. J Neurotrauma. 2007, 24:1687-97. 10.1089/neu.2007.0343

33. Prodam F, Gasco V, Caputo M, et al.: Metabolic alterations in patients who develop traumatic brain injury (TBI)-induced hypopituitarism. Growth Horm IGF Res. 2013, 23:109-13. 10.1016/j.ghir.2013.04.001

34. Blair JC: Prevalence natural history and consequences of posttraumatic hypopituitarism: a case for endocrine surveillance. Br J Neurosurg. 2010, 24:10-17. 10.3109/02688690903536637

35. Kreitschmann-Andermahr I, Poll EM, Reineke A, et al.: Growth hormone deficient patients after traumatic brain injury--baseline characteristics and benefits after growth hormone replacement--an analysis of the German KIMS database. Growth Horm IGF Res. 2008, 18:47278. 10.1016/j.ghir.2008.08.007

36. High WM Jr, Briones-Galang M, Clark JA, et al.: Effect of growth hormone replacement therapy on cognition after traumatic brain injury. J Neurotrauma. 2010, 27:1565-75. 10.1089/neu.2009.1253

37. Agha A, Phillips J, O'Kelly P, Tormey W, Thompson CJ : The natural history of post-traumatic hypopituitarism: implications for assessment and treatment. Am J Med. 2005, 118:1416. 10.1016/j.amjmed.2005.02.042

38. Reifschneider K, Auble BA, Rose SR.: Update of endocrine dysfunction following pediatric traumatic brain injury. J Clin Med. 2015, 4:1536-60. 10.3390/jcm4081536

39. Rosario ER, Aqeel R, Brown MA, Sanchez G, Moore C, Patterson D: Hypothalamic-pituitary dysfunction following traumatic brain injury affects functional improvement during acute inpatient rehabilitation. J Head Trauma Rehabil. 2013, 28:390-96. 10.1097/HTR.0b013e318250eac6

40. Popovic V, Aimaretti G, Casanueva FF, Ghigo E: Hypopituitarism following traumatic brain injury. Growth Hormone Deficiency in Adults. Jørgensen JOL, Christiansen JS (ed): Karger, Basel; 2005. 33:33-44. 10.1159/000088399

41. Zaben M, El Ghoul W, Belli A: Post-traumatic head injury pituitary dysfunction . Disabil Rehabil. 2013, 35:522-25. 10.3109/09638288.2012.697252 